\title{
VERZEICHNIS DER LITERATURWISSENSCHAFTLICHEN HABILITATIONEN AN ÖSTERREICHISCHEN UNIVERSITÄTEN
}

\author{
Vorbemerkung der Redaktion
}

Ab 1985 bringen wir das ,Verzeichnis in jedem zweiten Halbband eines Jahrganges. Für diese Dokumentation laden wir neu Habilitierte ein, ihre Anzeige an die Redaktion 'Sprachkunst (Österreichische Akademie der Wissenschaften, Institut für Kulturwissenschaften und Theatergeschichte, Postgasse 7/1/1, 1010 Wien) jeweils bis spätestens Ende Juli zu schicken. Folgende Angaben werden erbeten: Name, Geburtsjahr, Beruf, Titel der Dissertation, Publikationsverzeichnis, Titel und Kurzfassung (ca 20 Zeilen/190 Wörter) der Habilitationsschrift, Venia, Institutszuordnung.

\section{Ao. Univ.-Prof. Dr. Zohra Bouchentouf-Siagh}

Geboren: 1950

B e r u f: Außerordentliche Universitätsprofessorin am Institut für Romanistik der Universität Wien.

Dissertationen:

- Le système phonologique de quelques parlers arabes de l'Ouest algérien (Ghazaouet, Oran, Tlemcen). DEA, Algier 1976, 195 S.

- 1984, Les usages linguistiques dans le théâtre amateur algérien (1978-1981). Thèse de troisième cycle, Université René Descartes, Paris 1984, V + 196 S. [Siehe Publikationen.]

Publikationen:

Monographien:

- Le système phonologique de quelques parlers arabes de l'Ouest algérien (Ghazaouet, Oran, Tlemcen). DEA, Algier 1976, 195 S.

- 1984, Les usages linguistiques dans le théâtre amateur algérien (1978-1981). Thèse de troisième cycle, Université René Descartes - Paris 1984, V + 196 S.

Herausgaben:

- Algérie des femmes. Approches littéraires et linguistiques (= Quo vadis Romania? ${ }^{\circ} 11$ ), Institut für Romanistik, Wien 1998.

- [Zus. mit P. Cichon:] Sénégal. (= Quo vadis Romania? nº 21), Institut für Romanistik, Wien 2003. 
- Dzayer, Alger. Ville portée, rêvée, imaginée. Lectures autrichiennes, Casbah Éditions, Algier 2006.

Aufsätze:

- [Zus. mit D. Morsly und L. Moussaoui:] Étoffes et soieries, in: Kalim, n² 2/3, Institut des langues étrangères, Université d'Alger, Office des Publications Universitaires (OPU), Algier 1981, pp. 16-29.

- Le théâtre amateur et son public, in: Kalim nº 4, Institut des langues étrangères, Université d'Alger, OPU, Algier 1982.

- Approche sémiologique des enseignes en Algérie: message iconique et message linguistique, in: Balades dans la culture en Algérie en 1979, OPU, Algier 1984, pp. 245-271.

- Effets de l'insécurité linguistique et rhétorique en situation plurilingue, in: Peuples méditerranéens $n^{\circ} 33$, Paris 1985 .

- Style vocal et expressivité, in: Langues et littératures. Revue de l'Institut des langues étrangères n 3, OPU, Algier 1988, pp. 128-138.

- Du côté des petites filles: néologie, jeux floraux et intégration sociale, in: Enfances de $A$ à $Z$ - Actes du onzième colloque de français - Université d'Alger, Revue de l'Institut des langues étrangères, OPU, Algier 1989, pp. 37-40.

- Le théâtre amateur en Algérie: choix et usage de langues, in: International Journal of the Sociology of Language $n^{\circ}$ 87: Sociolinguistics of the Maghreb, Mouton de Gruyter, Berlin und New York 1990, pp. 71-86.

- La politique linguistique algérienne de 1985 à 1989, in: Th. Bulot/G. V. Martin (Hrsgg.), Sociolinguistique et didactique du FLE. Deux domaines en mutation, SUDLA, Université de Rouen 1990, pp. 89-99.

- Interrogations autour du théâtre de Kateb Yacine, Actes du Colloque international Kateb Yacine, OPU, Algier 1990, pp. 335-340.

- „Palestine trahie“: rhétorique d'un fauteuil roulant, in: N. KHAdDA (Hrsg.), Kateb Yacine et la modernité textuelle, OPU, Algier 1992.

- Hassiba et Aïcha: le vécu du mot „arabe“ en Algérie, in: Mots n 30. Les langages du politique, Presses de la fondation nationale des sciences politiques, Paris 1992, pp. 102-104.

- Le français en Algérie. Aspects de la politique scolaire coloniale (1830-1962), in: Quo vadis Romania?, ${ }^{\circ} 1$, Institut für Romanistik, Wien 1993, pp. 37-44.

- Éloge du mélange, in: Quo vadis Romania? n³ 3nstitut für Romanistik, Wien 1994, pp. 39-45.

- Le théâtre algérien, un aspect de la modernité?, in: N. Khadda (Hrsg.), Écrivains maghrébins et modernité textuelle. Études littéraires maghrébines nº 3, Paris: L'Harmattan 1994, pp. 73-86.

- [Zus. mit J. Zwobada-Rozel:] Mise en place d'une formation universitaire dans un pays en voie de développement (Algérie 1964-1981), in: C. Labat/G. Vermès (Hrsgg.), Cultures ouvertes, sociétés interculturelles, Paris: L'Harmattan 1994, pp. 304-313.

- Frauen des Maghreb, die voranschreiten (Übersetzung von Hildegard Hefel), in: Frauensolidarität $n^{\circ}$ 50, Wien 1994, p. 7.

- Syntaxe et vision du monde, in: N. Khadda (Hrsg.), L'honneur de la tribu de R. Mimouni. Lectures algériennes. Études littéraires maghrébines nº 4, Paris: L’Harmattan 1995, pp. 86-92.

- Le minoritaire sur la scène: femmes et langues populaires au théâtre (Algérie, 19881992), in: Quo vadis Romania? n 6, Institut für Romanistik, Wien 1995, pp. 67-75. 
- Catégorie nomade: le prénom en arabe algérien, lexème ou déictique?, in: P. Cichon et. al. (Hrsgg.), Lo gai saber. Zum Umgang mit sprachlicher Vielfalt. Festschrift für Georg Kremnitz, Wien: Braumüller 1995, pp. 303-310.

- Notes sur le théâtre algérien, in: C. Bonn/N. Khadda/A. Mdarhri-Alaoui (Hrsgg.), Littérature maghrébine d'expression française, EDICEF/AUPELF, Paris 1996, pp. $136 \mathrm{f}$.

- Aspects de la réalité culturelle et linguistique française d'aujourd'hui, à travers quelques romans dits „beurs“, in: Cahiers francophones d'Europe centre-orientale n ${ }^{\circ}$ 5-6, t. II, Pécs, Wien 1996, pp. 315-326.

- Le hammam, espace de parole et signe urbain, in: N. Khadda/P. Siblot (Hrsgg.), Alger - une ville et ses discours, Praxiling, Université Paul Valéry, Montpellier 1996, III, pp. 311-319.

- Les langues populaires au Maghreb entre glottopolitique et revendications identitaires, in: P. Cichon (Hrsg.), Das sprachliche Erbe des Kolonialismus in Afrika und Lateinamerika, Wien: Edition Praesens 1996, pp. 23-30.

- Unterdrückung von Frauen im Spiegel der Bühne. Theater des Widerstands in Algerien (Übersetzung von Hildegard Hefel), in: Frauensolidarität n ${ }^{\circ} 56$, Wien 1996, pp. 5 f.

- Kader, l'homme de parole(s), in: En mémoire du futur. Pour Abdelkader Alloula (Sammelband), Actes Sud, Aix-en-Provence 1997, pp. 37-40.

- Frauenliteratur in Afrika (Zusammenfassung eines Vortrags, Deutsch von Hildegard Hefel), in: Frauensolidarität n ${ }^{\circ}$ 61, Wien 1997, pp. $22 f$.

- Parallelen zum Faschismus. Im Gespräch mit Khalida Messaoudi, (in Zusammenarbeit mit Hildegard Hefel), in: Frauensolidarität nº 62, Wien 1997, pp. 26.

- Migrants et choix de sépulture en terre d'accueil: moment zéro de l'„intégration“?, in: C. Chaulet-Achour (Hrsg.), Cahiers Jamel Eddine Bencheikh. Savoir et imaginaire, Études littéraires maghrébines n ${ }^{13}$, Paris: L'Harmattan 1998, pp. 149-157.

- Littérature et expressions féminines (Vorstellung der Sondernummer), in: Quo vadis Romania? nº 11, Institut für Romanistik, Wien 1998, pp. 5 f.

- Une institutrice parle ... une liberté au goût de coing. Raconter sa ville (Alger) en interaction verbale, in: Quo vadis Romania? n 11, Institut für Romanistik, Wien 1998, pp. 64-73.

- Das verlorene Alphabet. Schreiben zwischen zwei Sprachen: Assia Djebar, (Übersetzung von Hildegard Hefel), in: Frauensolidarität n 64, Wien 1998, pp. $7 f$.

- Mimer la langue absente: rhétorique et colinguisme dans quelques romans de l'immigration, in: E. RuHe (Hrsg.), Die Kinder der Immigration - Les enfants de l'immigration. Studien zur Literatur und Geschichte des Maghreb, Band 4, Würzburg: Königshausen $\&$ Neumann, 1999, pp. 83-94.

- L'Algérie: quelques données [informative Texte über Algerien, mit Impulsen zu ihrer didaktischen Verwertung und mit einer Mini-Anthologie], in: M. EIsL, Tour d'horizon 2000, tome 3 [Französisch-Lehrbuch für AHS und BHS, die Sekundarstufen in Österreich], Salzburg: Verlegergemeinschaft Neues Schulbuch 2000, pp. 138.

- Camus le baliseur [im Jahr 2000 im Rahmen der „Wiener Vorlesungen“ gehaltener Vortrag, in der deutschen Übersetzung durch BIRGIT WAGNER erschienen mit dem Titel 'Markierungen im Gelände. Camus in Afrikał], in: Z. Bouchentouf-Siagh/P. Kampits, Zur Aktualität von Albert Camus, Wien: Picus 2001, pp. 22-45.

- Expériences didactiques et enseignement de la culture, in: M. Bedjaour (Hrsg.), Langues, cultures et traduction. Cahiers de traduction. Actes du Colloque de l'Université d'Alger - 11-12.4.2001, Les Éditions El Hikma, Algier 2001, pp. 12-24. 
- Cacophonie et brouillage du récit dans „La Princesse et le clown“ de Hamid Skif: la fiction impossible?, in: B. Burtscher-Bechter/B. Mertz-Baumgartner (Hrsgg.), Subversion du réel: stratégies esthétiques dans la littérature algérienne contemporaine, Études Littéraires Maghrébines n 16, Paris: L'Harmattan 2001, pp. 27-40.

- Le coup de force de l'étrangère. Poème (asefrou) berbère sur la langue française, in: Сн. Адоваті et al. (Hrsgg.), Wenn Ränder Mitte werden. Zivilisation, Literatur und Sprache im interkulturellen Kontext. Festschrift für F. Peter Kirsch, WUV, Wien 2001, pp. 378-388.

- Une lecture africaine de Gide. „Le Voyage au Congo suivi de Le retour du Tchad“, in: I. Albers/A. Pagni/U. Winter, Blicke auf Afrika nach 1900 - Französische Moderne im Zeitalter des Kolonialismus. XXVI. Romanistentag in Osnabrück 1999, Tübingen: Stauffenburg 2002, pp. 105-116.

- „Les Paravents“ (Genet), „Mille Hourras pour une gueuse“ (Dib). Scénographie comparée, in: N. KhadDa (Hrsg.), Mohammed Dib. 50 ans d'écriture, Université Paul Valéry, Montpellier 2002, III, pp. 143-152.

- Lob der Mischung [deutsche Übersetzung durch Reinhart Hosch von „Éloge du mélange" (1994)], in: B. Czernilofsky/G. Kremnitz, Trennendes. Verbindendes. Selbstzeugnisse zur individuellen Mehrsprachigkeit, Wien: Edition Praesens 2003, pp. 89-95.

- Interview avec Alioune Badara Diop: un exemple de plurilinguisme africain, in: Quo Vadis Romania? $n^{\circ} 21$, Institut für Romanistik, Wien 2003, pp. 90-102.

- Tamazight au Maghreb: la „longue durée“. Aspects glottopolitiques en Algérie, in: Quo Vadis Romania? n ${ }^{\circ} 22$, Institut für Romanistik, Wien 2003, pp. 84-94.

- D'Albert Camus à Roland Barthes, une lecture politique des signes, in: S. BrodziAK/CH . Chaulet-Achour et al. (Hrsgg.), Albert Camus et les écritures du XXe siècle. Actes du Colloque International de l'Université Cergy-Pontoise - novembre 2001, Artois Presses Université, Université Cergy-Pontoise 2003, pp. 201-209.

- 'Ici - là-bas': la trame de soi dans „La Transe des insoumis“ de Malika Mokeddem, in: A. Bererhi (Hrsg.), L'autobiographie en situation d'interculturalité. Actes du Colloque de l'Université d'Alger, t. II, Éditions du Tell, Blida 2004, pp. 331-338.

- En guise d'introduction: ceci n'est pas un livre sur Alger, in: Z. Bouchentouf-Siagh (Hrsg.), Dzayer, Alger. Ville portée, rêvée, imaginée. Lectures autrichiennes, Casbah Éditions, Algier 2006, pp. 7-12.

- A. Camus et le refoulé colonial: une lecture de `Le Minotaure ou la halte d'Oran', in: A. Bererhi (Hrsg.), Albert Camus et les Lettres Algériennes: l'espace de l'inter-discours, Actes du Colloque international de Tipaza-Alger (24.-28. April 2006), Université d'Alger - Département de Français, t. I, Éditions du Tell, Blida 2007, pp. 135-144.

- Boubacar Boris Diop: une poétique de la dissidence, in: Actes du Colloque international sur les littératures postcoloniales, Tamanrasset (April 2007), Éditions du Tell, Blida 2007, pp. 167-176.

- Duplicité et trafic de l'Histoire, in: Makhily Gassama (Hrsg.), Contre le discours de Dakar. L'Afrique répond à Nicolas Sarkozy, Philippe Rey, Paris 2008, pp. 53-76.

- Échanges méditerranéens au théâtre. Quelques repères pour comprendre le présent, in: Сн. Chaulet-Achour (Hrsg.), Itinéraires intellectuels entre la France et les rives sud de la Méditerranée, Éditions Khartala, Paris 2009, pp. 193-205.

- Robert Laffont l'Occitan, Mouloud Mammeri le Kabyle: deux „voleurs de feu“? Amusnaw l'un comme l'autre?, in: G. Latry, (Hrsg.), La Voix occitane. Actes du VIIIe Colloque 
de l'AIEO - Bordeaux, 12-18.9.2005, tome 2, Presses universitaires de Bordeaux, Pessac 2009, pp. 1417-1429.

- Imaginaire linguistique et production littéraire francophone. Le cas de trois écrivaines algéro-françaises: Assia Djebar, Malika Mokeddem et Leïla Sebbar, in: Musanjı NGALASSO-Mwatha (Hrsg.), Imaginaire linguistique dans les discours littéraires, politiques et médiatiques en Afrique. Actes du Colloque International de l'Université M. de Montaigne de Bordeaux - 10-13.12.2008, Presses universitaires de Bordeaux, Pessac 2010, pp. 225-240.

- Déplacement des lignes et inscription de la trace dans l'œuvre de Malika Mokeddem, in: A. BeKat/A. Bererhi/B. Lebdaï (Hrsgg.), Écritures nomades. Actes du Colloque International de Djanet - 11-15.4.2009, Éditions du Tell, Blida 2010, pp. 63-68.

- Parole de maghrébine, in: Makhily Gassama (Hrsg.), 50 ans après - quelle indépendance pour l'Afrique?, Philippe Rey, Paris 2010, pp. 69-83.

- Histoire, identité et imaginaire dans l'œuvre de Maïssa Bey, Colloque International de Sousse (Tunesien), 15.-17.4.2010 [im Druck].

- Une femme à la traversée du siècle: „Histoire de la femme cannibale“ de Maryse Condée, Colloque international d'Alger, 12.-14.12.2010 [im Druck].

- Questions posées à la sociolinguistique (maghrébine) par la transcription du tamazight au Maghreb. État des lieux, Internationales Symposium „Les politiques linguistiques explicites et implicites en domaine francophone“, Wien 14.-15.10.2011 [im Druck].

Habilitationsschrift:

Paroles maghrébines, Wien 2010, 542 Seiten.

Kurzfassung:

Der Titel >Paroles maghrébines` versteht sich einerseits als politischer Anspruch, andererseits als epistemologisches und wissenschaftliches Programm im postkolonialen Kontext. Richtlinie bei wirklich jeder Themenstellung war dabei das geschichtsbewusste Epistemologieverständnis Pierre Bourdieus: „Wer beansprucht zu wissen, was er tut, wenn er Wissenschaft betreibt, sollte wissen, wie die jeweiligen Probleme, Instrumente, Methoden und Konzepte zu dem gemacht wurden, was sie heute für ihn sind“ (Pierre Bourdieu). >Paroles maghrébines bezeugt eine fortlaufende Forschung, deren Theorie-Ansatz sich am Prinzip der Komplexität und Kohärenz insofern orientiert, als er in der Unterrichtssituation und an den verschiedensten Aspekten des Sprachbereichs erprobt wird: reine Linguistik etwa in Form von Untersuchungen zu den arabischen Dialekten, angewandte Linguistik bei der Sprecherziehung sprachgestörter Patienten, Soziolinguistik als Disziplin, die im nordafrikanischen und französischen wie frankophonen Kontext politische Bedingtheiten mit ihren Sprachverboten und Sprachkonflikten untersucht. Sprache ist indes nicht nur Kommunikationsmittel, sondern auch ein semiotisches System unter vielen, das einzige allerdings, das eine Metasprache generiert, mit der andere semiotische Systeme analysierbar werden (Saussure, Peirce, Hjelmslev); daher auch die vorliegenden Analysebereiche der kulturellen Hervorbringungen (in einem weit gefassten Kulturbegriff) sowie der Theaterpraxis und der französischen und frankophonen Literaturen. In >Paroles maghrébines` wird aus einem ethisch und epistemologisch ausgerichteten Wissenschaftsblickwinkel das sozio-kulturelle Feld Algeriens und des Maghreb betrachtet und in weiterer Folge das Projekt einer wirklich entkolonialisierten maghrebinischen Gesellschaft entworfen, die sich vermeintlicher Entscheidungszwänge bewusst wird und sich von ihnen nicht unterjochen lässt. Auf diese 
Weise wird mit Hilfe des Konzepts einer Maghrébinité (in freilich unterschiedsbewusster Anlehnung an den Begriff der Créolité) versucht, sozio-kultureller Vielfalt gerecht zu werden und sie wirkkräftig bewusst zu machen.

Venia:

Französische Sprach-, Literatur- und Landeswissenschaft.

Institutszuordnung:

Institut für Romanistik der Universität Wien.

Univ.-Doz. Mag. Dr. Ioannis Zelepos

Geboren: 1967

B er u f: Univ.Doz. am Institut für Geschichte der Universität Regensburg; Lehrbeauftragter am Institut für Byzantinistik und Neogräzistik der Universität Wien.

Dissertation:

Die Ethnisierung griechischer Identität 1870-1912. Staat und private Akteure vor dem Hintergrund der ,Megali Idea', München 2002, 300 S. [Siehe Publikationen.]

Publikationen:

Monographien:

- Erzbischof Makarios III. von Zypern und die ,Enosis'. Kontinuität und Wandel in der Politik der Vereinigung Zyperns mit Griechenland von 1950 bis 1974, Hamburg 1994. (Magisterarbeit FB Geschichtswissenschaften der Universität Hamburg.)

- Die Ethnisierung griechischer Identität 1870-1912. Staat und private Akteure vor dem Hintergrund der ,Megali Idea'. Südosteuropäische Arbeiten Bd. 113, München: Oldenbourg-Verlag 2002. (Dissertation.)

- Rebetiko. Die Karriere einer Subkultur, Köln: Romiosini-Verlag Köln 2001.

- Die Kollyvadenbewegung. Zu den Auseinandersetzungen um Tradition, Aufklärung und Identität im osmanisch-orthodoxen Kommunikationsraum 1750-1820 (= Südosteuropäische Arbeiten), München: Oldenbourg-Verlag 2012. (Habilitationsschrift.)

Herausgaben:

- [Zus. mit Maria A. Stassinopoulou:] Griechische Kultur in Südosteuropa in der Neuzeit. Beiträge zum Symposium in memoriam Gunnar Hering (Wien, 16.-18. Dezember 2004) (= Byzantina et Neograeca Vindobonensia Bd. XXVI), Wien: ÖAW 2008.

- [Zus. mit Ulrike Tischler:] Bilderwelten - Weltbilder. Die Gegenwart der Vergangenheit in postosmanischen Metropolen Südosteuropas [Thessaloniki, Istanbul, Izmir], Frankfurt/M.: Peter Lang 2010. 
- [Zus. mit Maria Oikonomou und Maria A. Stassinopoulou:] Griechische Dimensionen südosteuropäischer Kultur seit dem 18. Jahrhundert (=Studien zur Geschichte Südosteuropas Bd. 17), Frankfurt/M.: Peter Lang 2011.

Aufsätze:

- Zeittafel der Geschichte Zyperns. in: Winfried Steffani/Peter Zervakis/K. D. Grothusen ( $\dagger$ ) (Hrsgg.), Südosteuropa-Handbuch Bd. VIII. Zypern, Göttingen: Vandenhoek \& Ruprecht 1998, S. 820-842.

- Die Insel Ikaria vom Juli bis November 1912. Der Aufstand gegen die osmanische Herrschaft und der, Freistaat Ikaria bis zur Vereinigung mit Griechenland, in: IoANNis Vassis/Günther S. Herich/Diether R. Reinsch (Hrsgg.), Lesarten. Festschrift für Athanasios Kambylis zum 70. Geburtstag, Berlin: de Gruyter 1998, S. 338-350.

- „Ägäis“ in: Holm Sundhaussen/Heiko Hänsel (Hrsgg.), Konfliktregionen Südosteuropas im Zeitalter des Nationalismus (Teil 2), Arbeitspapiere des Osteuropa-Instituts der FU Berlin, 4/2001, S. 60-66.

- „Zypern“ in: Holm Sundhaussen/Heiko Hänsel (Hrsg.), Konfliktregionen Südosteuropas im Zeitalter des Nationalismus (Teil 2), Arbeitspapiere des Osteuropa-Instituts der FU Berlin, 4/2001, S. 67-72.

- Griechische Händler und Fanarioten in Süd- und Südosteuropa von der Frühen Neuzeit bis zum 19. Jahrhundert, in: Klaus J. Bade/Pieter C. Emmer/Leo Lucassen/ Jochen Oltmer (Hrsgg.), Enzyklopädie. Migration in Europa. Vom 17. Jahrhundert bis zur Gegenwart, München: Schöningh 2007, S. 615ff. (Engl. u. d. T.: Greek Traders and Phanariotes in Southern and Southeastern Europe from the Early Modern Period to the Nineteenth Century, in: Klaus J. Bade/Pieter C. Emmer/Leo Lucassen/ Jochen Oltmer (Eds.), The Encyclopedia of Migration and Minorities in Europe. From the 17th Century to the Present, Cambridge 2011, S. 472-473.)

- Griechische Siedler im Schwarzmeerraum in Neurussland seit der frühen Neuzeit und Pontosgriechen in Griechenland seit dem Zweiten Weltkrieg in: Klaus J. Bade/ Pieter C. Emmer/Leo Lucassen/Jochen Oltmer (Hrsgg.), Enzyklopädie. Migration in Europa. Vom 17. Jahrhundert bis zur Gegenwart, München: Schöningh 2007, S. 617-622. (Engl. und. d. T.: Greek Settlers from the Black Sea Region in New Russia since the Early Modern Period and Pontic Greeks in Greece since the End of World War II, in: Klaus J. Bade/Pieter C. Emmer/Leo Lucassen/Jochen Oltmer (Eds.), The Encyclopedia of Migration and Minorities in Europe. From the 17th Century to the Present, Cambridge 2011, S. 468-472.)

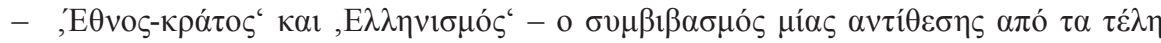

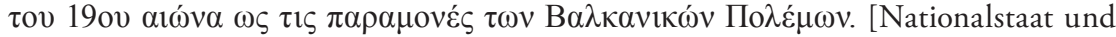
„Hellenismus" - zur Vereinbarung eines Widerspruchs in der Zeit vom Ende des 19.

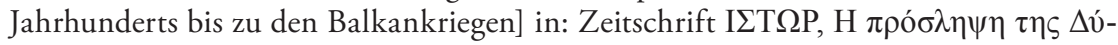

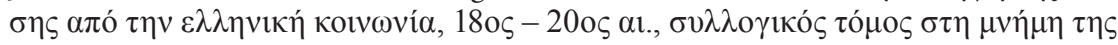
'Е $\lambda \lambda \eta \varsigma$ $\Sigma \kappa о \pi \varepsilon \tau \varepsilon \dot{\alpha}$. [Im Druck.]

- ,Phoenix ohne Asche'. Konstantinos Paparrigopoulos und die Entstehung einer griechischen Nationalhistoriographie im 19. Jahrhundert, in: Markus Krzoska/Hans-Christian Maner (HrsG.), Beruf und Berufung. Geschichtswissenschaft und Nationsbildung in Ostmittel- und Südosteuropa im 19. und 20. Jahrhundert (= Studien zur Geschichte, Kultur und Gesellschaft Südosteuropas; Bd. 4), Münster: LIT-Verlag 2005, S. 190-215. 
- Rebetiko. Anmerkungen zu einem schillernden Begriff. in: Zeitschrift PHILIA/2005 II, S. 72-98.

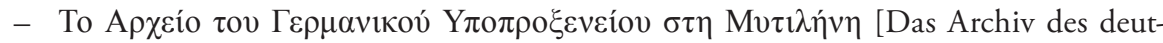

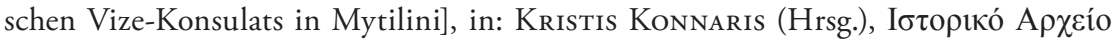

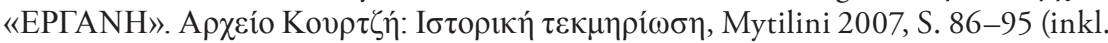
2 CD-ROM mit digitalen Datenbanken).

- Projektionen des Authentischen. Zur Bedeutung von Makrygiannis für die neugriechische Literatur, in: Konstantina Glykioti/Doris Kinne (Hrsgg.), Griechisch -

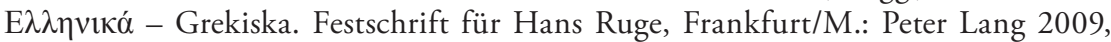
S. 245-251.

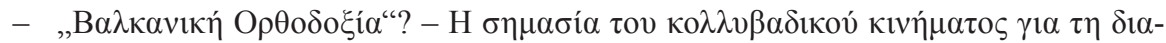

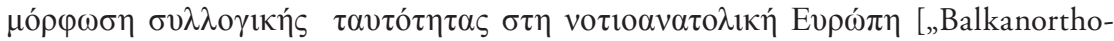
doxie"? - Zur Bedeutung der Kollyvadenbewegung für die Ausbildung kollektiver

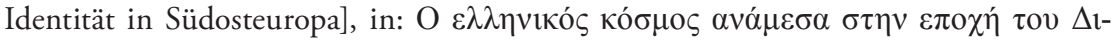

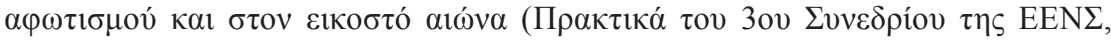

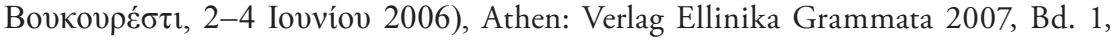
S. 511-523 (auch unter http://www.eens.org/start.asp).

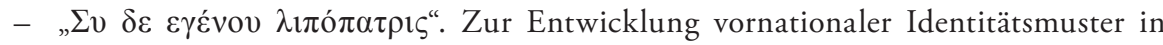
Südosteuropa: der ,osmanisch-orthodoxe‘ Heimatbegriff von Michailos Perdikaris (1766-1828), in: Ulf Brunnbauer/Andreas Helmedach/Stefan Troebst (Hrsgg.), Schnittstellen. Gesellschaft, Nation, Konflikt und Erinnerung in Südosteuropa. Festschrift für Holm Sundhaussen zum 65. Geburtstag (= Südosteuropäische Arbeiten; Bd. 133), München: Oldenbourg-Verlag 2007, S. 189-200.

- Ioannis Makrygiannis, ,Apomnimonevmata' (Memoiren) - Biogramm und Werkartikel für Kindlers Literatur Lexikon, 3., vollständig neu überarbeitete Auflage 2009.

- Ion Dragoumis, ,Martyron kai iroon aima' (Blut von Märtyrern und Helden) - Biogramm und Werkartikel für Kindlers Literatur Lexikon, 3., vollständig neu überarbeitete Auflage 2009.

- Städte als Projektionsflächen im griechischen Popularlied des 20. Jahrhunderts am Beispiel Istanbuls, Izmirs und Thessalonikis, in: Ulrike Tischler/Ioannis Zelepos (Hrsgg.), Bilderwelten - Weltbilder. Die Gegenwart der Vergangenheit in postosmanischen Metropolen Südosteuropas [Thessaloniki, Istanbul, Izmir], Frankfurt/M.: Peter Lang 2010, S. 63-100.

- Amateurs as nation builders? On the significance of associations for the formation and nationalization of the greek society in the $19^{\text {th }}$ century, in: Hannes Grandits/ Natalie Clayer/Robert Pichler (Hrsg.), Conflicting Loyalties in the Balkans. The Great Powers, the Ottoman Empire and Nation Building, London: Tauris 2011, S. 64-85.

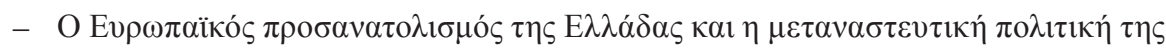
$\kappa v \beta \varepsilon ́ \rho v \eta \sigma \eta \varsigma$ K $\alpha \rho \alpha \mu \alpha \nu \lambda \eta \dot{~ \sigma \tau \eta v ~ о к \tau \alpha \varepsilon \tau i ́ \alpha ~ ' 55-' 63 ~[T h e ~ e u r o p e a n ~ o r i e n t a t i o n ~ o f ~ G r e e c e ~}$ and the migration policy of the Karamanlis-government in the years 1955-1963] in: Konstantinos Svolopoulos, Konstantina Botsiou, Evanthis Hatzivassiliou (Hrsgg.), Konstantinos Karamanlis in the Twentieth Century, Conference, Zappeion, Athens 5-9 June 2007, Bd. 3, Athen: Konstantinos Karamanlis Foundation 2008, S. 113-123.

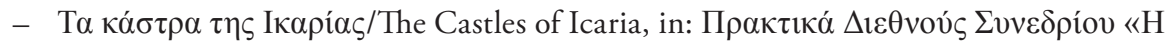




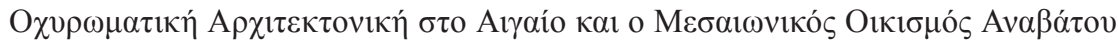
Xíov», 26-28 $\Sigma \varepsilon \pi \tau \varepsilon \mu \beta$ píov 2008, Mytilini 2011, S. 171-182.

- Nationsbildung und Nationalismus in Griechenland, in: BJörn Egner/Georgios Terizakis (Hrsgg.), Das politische System Griechenlands - Strukturen, Akteure, Politikfelder, Baden Baden: Nomos 2009, S. 39-59.

- Politische Lieder - Lieder als Politikum. Das Beispiel Griechenlands im 20. Jahrhundert, in: Stefan Michael Newerkla/Fedor Poljakov/Oliver Jens Schmitt (Hrsgg.), Das politische Lied in Ost- und Südosteuropa (= Europa Orientalis; Bd. 11), Wien: LIT-Verlag 2010, S. 29-47.

- „Unser orientalisch-christliches Geschlecht“ - Zur Formierung eines osmanisch-orthodoxen Identitätskonzepts in der zweiten Hälfte des 18. Jahrhunderts, in: Maria Oikonomou/Maria A. Stassinopoulou/Ioannis Zelepos (Hrsgg.), Griechische Dimensionen südosteuropäischer Kultur seit dem 18. Jahrhundert. Beiträge zum Symposium (Wien, 17.-18. Dezember 2009) (= Studien zur Geschichte Südosteuropas; Bd. 16), Frankfurt/M.: Peter Lang 2011, S. 111-124.

- Griechenland, in: Peter Brandt/Werner Daum (Hrsgg.), Handbuch der europäischen Verfassungsgeschichte im 19. Jahrhundert, Teil 2 (1815-1847). [Im Druck.]

- Vampirglaube und Orthodoxe Kirche im osmanischen Südosteuropa. Ein Fallbeispiel für die Ambivalenzen vorsäkularer Rationalisierungsprozesse, in: Das osmanische Europa. Methoden und Perspektiven der Frühneuzeitforschung zu Südosteuropa. [Im Druck.]

\section{Mitwirkung an Publikationen:}

- Richard ClogG, Geschichte Griechenlands im 19. und 20. Jahrhundert, Köln: Romiosini 1998. [Lektorat der deutschen Übersetzung; Korrektur von inhaltlichen Ungenauigkeiten im englischen Original von 1992; Ergänzungen aktueller Entwicklungen im Zeitraum von 1992-1998.]

- Elias Petropoulos, Rebetiko. Die Musik der städtischen Subkultur Griechenlands, Heidelberg: Palmyra 2002 (inkl. Audio-CD). [Mitwirkung bei der Übersetzung der Liedertexte; Auswahl des Bildmaterials und der Beispiellieder; Ergänzungen zur Bibliographie und zu weiterführenden Links.]

\section{Rezensionen:}

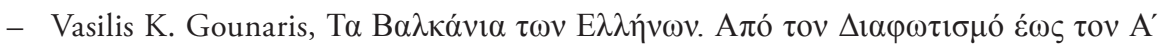

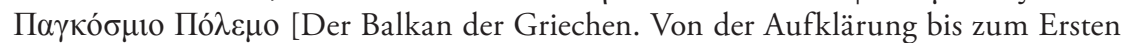
Weltkrieg], in: Südost-Forschungen Bd. 68/2009, S. 556-559.

- Heinz A. Richter, Geschichte der Insel Zypern. Band IV: 1965-1977, in: SüdostForschungen Bd. 68/2009, S. 698-700.

Habilitationsschrift:

Die Kollyvadenbewegung. Zu den Auseinandersetzungen um Tradition, Aufklärung und Identität im osmanisch-orthodoxen Kommunikationsraum 1750-1820 (= Südosteuropäische Arbeiten), München: Oldenbourg-Verlag 2012, 430 S. [Siehe Publikationen.]

Kurzfassung:

Epigramme spielen innerhalb des literarischen Schaffens der Byzantiner eine wichtige Rolle. In nicht geringer Anzahl (mehr als 1000 Stück) sind diese auch heute noch in- 
schriftlich erhalten, sei es auf Fresken, Mosaiken, Ikonen, auf so genannten Objekten der Kleinkunst und in Handschriften, in denen sie als Rahmen von Miniaturen fungieren oder Figurengedichte bilden. Die vorliegende Studie widmet sich Epigrammen auf Fresken und Mosaiken. Aus dem Untersuchungszeitraum (600 n. Chr.-1500 n. Chr.) sind mehr als 250 Epigramme auf Fresken und knapp zwanzig Epigramme auf Mosaiken überliefert. In einer ausführlichen Einleitung wird auf Fragen der Definition, der Geschichte und der äußeren Gestaltung byzantinischer Epigramme ebenso wie auf die Besonderheiten byzantinischer Epigramme auf Fresken und Mosaiken, so z. B. auf die Interaktion von Wort und Bild, eingegangen. Im Hauptteil der Arbeit folgen die kritische Edition der Epigramme, deren deutsche Übersetzung und ein philologisch-sprachlicher sowie historisch-realienkundlicher Kommentar. Indices zu Epigrammanfängen, griechischen Wörtern, biblischen, antiken und byzantinischen Stellen und ein allgemeiner Index zu Orten und Personen runden den Band ab. Zur optischen Veranschaulichung sind fast alle behandelten Epigramme auch bildlich dokumentiert.

Venia:

Südosteuropäische Geschichte und Neogräzistik.

Institutszuordnung:

Historisch-Kulturwissenschaftliche Fakultät der Universität Wien (Institut für Byzantinistik und Neogräzistik, Institut für Osteuropäische Geschichte). 